\title{
Planning and Implementation: Towards an 'Open' Approach
}

\section{A. S. Barnett}

\section{Introduction}

Plans, programmes and policies are evolved and implemented by people in social relationships with each other. They are not the result of purely tech. nical appraisals and decisions. In particular, they are developed and implemented through formal organizations such as ministries and other government agencies. It is frequently the case that the ideological approach of the government agency, together with its mode of operation, may in effect contradict the aims of a plan. For example, an organization which attempts to bring about cooperation may be ill-suited to that task if its own style and structure is very authoritarian and hierarchical. It is, indeed, a common paradox that bureaucracies are frequently expected to be able to organize such non-bureaucratic forms of behaviour as mutuality, communality and cooperation.

Policies may not be realized in practice for a number of reasons. One is that there may be explicit political opposition to them. Another is that the way in which the policy is being implemented may itself militate against its success. While the first type of obstruction is relatively easy to identify and is part of the explicit political process, the latter is less easily identified, and may form part of the covert, broader political process, or may be unintended. Little attention has been paid to this aspect of plan formulation and implementation, yet the organizational structure, together with the style and assumptions which form the context for formulation and implementation of plans, are not neutral. They all carry messages both to the planners and to those whom the plans will affect directly about the way the world is and ought to be.

\section{The Planning Process: a Characterization}

Both planning and implementation tend to be processes in which people are directed as though they were resources rather than consulted as conscious agents who have wills, desires and needs. Planning decisions occur at central, regional and local levels, but almost always in the context of government. The ultimate consumers, the members of the local community, see and experience the final result-the plan-as something which has its origins outside themselves and their local communities. This experience of the 'otherness' of the plan and its implementation arises from a number of sources, some internal to the process of planning, others external. Among the latter, one factor which is obviously of major importance is the broader political context in which planning takes place. A plan is many things: it is a charter; it is in some contexts a discussion document; it is also a directive. Usually it attempts to achieve multiple goals. Some of these are stated explicitly as policy choices, but others are unstated, being the goals of particular groups within the political spectrum which affects a particular society. It is those sources of 'otherness' which arise from within the planning process itself that are identified for discussion here. The three major sources are the hierarchical context, the assumption of technique, and the 'as if' view of the world. Each of these can be explored in turn. (i) The hierarchial context. Planning and project implementation take place in a bureaucratic environment, usually in ministerial bureaucracies. The Civil Service is a vital part of the state machine and thus has more power and more authority than other non-state bureaucracies. In addition, in ex-colonial societies, there are historical reasons why government administration, particularly at the point of contact with clients, may be more impermeable to their demands. In the colonial situation, the administrative state was foreign, and although at its lowest reaches it was manned by indigenes, they were often put into contradictory positions where their loyalties were severely strained. Because of this and other reasons, for example racial attitudes on the part of their superiors, they were the objects of strict rules concerning the manner in which they were expected to deal with their clients. Administrators working in the post-colonial state within this historical tradition may tend towards perceptions of authority rather than communication -activeness rather than reactiveness. In short, they will behave and will be experienced as 'other' than their clients, and this will be reinforced by class and other differences. In these situations, initiative and original thought will be either completely stifled or discounted as not fitting into the existing rules. At the same time, authority is used as an excuse whereby the local level implementor of a plan blames his own lack of ini- 
tiative on the higher levels of the administrative machine.

(ii) The assumption of technique. Analytical techniques are not neutral objects but cultural and social products derived in the main from two sources. One is economic theory, particularly those areas concerned with cost-benefit analysis, linear programming, and input-output analysis; the second is the structure of administration itself. The use of techniques derived from economics is related to the terms of reference and the body of conventional wisdom enshrined within an administration. Thus, what is put through a particular technique is related to what is considered relevant by the administration which is attempting to use that technique, and these planning techniques embody particular perspectives on the world. Seen as cultural products, they are produced, reinforced and adapted by specific social groups and historical situations. Planning theory and action has by and large been the possession of the more educated groups, both local and foreign-the Mandarins.

In addition, there are the assumptions made by such groups in the course of planning about the nature of 'development': "Quantitative planning has exercised what amounts to methodological imperialism largely because no useful way existed for incorporating other elements into decisions about goals or the allocation of resources." Here, the goals of a particular project or programme are subverted by the techniques of administration and the categories of perception which inform the process in a pronounced and crucial form. The techniques, concepts and methods employed by planners inevitably reinforced the social and cultural hiatus between the planners and the planned, the implementer and his clients. The control of knowledge and techniques, their embodiment in a document called the plan and its specific projects, reinforces and demonstrates the pre-existing social, cultural, political and economic gaps between social groups. Thus, once more, the plan appears, and is experienced by the consumer (or the victim) as something outside himself.

(iii) The 'as if' view of the word. It is a cliché to say that one reason for making plans is in order to break them, but what this tells us is that planning assumptions are only approximations, scheduling only a guideline, and that these assumptions and guidelines present an 'as if' view of the world for the planner and administrator. In reality, however, they become constraints on

\footnotetext{
1 D. Goulat, The Cruel Chotce: a New Concept in the Theory of Development, Atheneum, New York, 1971 .
}

other sectors, both within planning and implementing agencies and, in a disaggregated form, on the broader population. Plans are formulated as directives concerning policy and timing. To the planner, who has an overall view, they may be clearly perceived as adaptable, but to those below they are understood and experienced as directives.

When the process begins to go astray, deadlines are not met, projects do not work, the reasons cannot be fully comprehended. Those who experience the failure of plans at the lower levels, do so only as they are mediated and interpreted through the planning culture, the administrative hierarchy and the conceptual apparatus of techniques. The world of 'as if' becomes the world of 'it is' and the failures and unanticipated consequences are experienced as personal or political failures, certainly not as the failure of a system, a world to which the client and the local administrator do not belong.

\section{The Planning Paradigm}

A common typification of the steps in planning might look as follows:

(i) identification of objectives-decisions about policies;

(ii) quantification of targets;

(iii) allocation of resources and decisions about phasing;

(iv) implementation;

(v) supervision;

(vi) evaluation.

But clearly this set of processes in its very nature is beset with problems. At each level, the reality involves a dimension of power or authority, mediated through the mechanisms of a government department. 'Identification', 'quantification', 'allocation', 'implementation', 'supervision' and 'evaluation' are all processes which can be less, or more commonly, more, authoritarian or participative. I am not suggesting that planning and implementation are either always coercive and authoritarian, or that they can necessarily be participative. It is rather that, as presently practised, the pressures are towards non-participation by the clients in all phases.

\section{Towards an 'Open' Approach}

The differences between the characterization presented above and the 'open' approach has parallels with models of science. One model sees science as logical, causal and experimenial; another sees the process as centred on paradigms which gain dominance and encourage research which fills out the detail. Planning, with its roots in economics and administration, is or pretends 
to be experimentally based; analytical and in some sense logical. The very steps in the typification presented above, from identification through to evaluation, echo the old incantation: theory, hypothesis, experiment, law. And yet we know that science does not follow this process, even though it is useful to act in some situations as though it did (where, for instance, scientific endeavour is concerned to fill out paradigms rather than invent new ones). But in planning and its implementation, we must always be creating new paradigms. A more useful approach might be borrowed from semantics, the study of meaning and communication of meaning ${ }^{2}$. We need to understand planning and plan implementation as an aspect of the communication of meaning, rather than as some kind of total logical, scientific and mechanical process. Concentration on processes of planning and implementation directs our attention away from the total field of meaning, of which the process may be only one part. It is in this guise that planning is often a mechanism of class or other forms of domination.

This point has something in common with the controversy between psychoanalysis and behavioural psychology. Rycroft argues that psychoanalysis is not an experimental science, but a semantic theory. It is the body of concepts which attempts to make sense of the experiences of human beings and to enable that experience to be communicated. Because of this quality, psychoanalysis should not pretend to meet the canons of experimental science. Indeed, he suggests that the controversy is largely the result of the two sides misunderstanding their own and each other's premises: "On their side the analysts are claiming that analysis is what it is not (an experimental science) and (on their side, their critics are) attacking it for failing to be what it has no need to claim to be". ${ }^{3}$

Similarly, the premises on which planning and implementation operate are perhaps misunderstood by some practitioners. The 'experimental' assumptions, imported from the premises of economics and administrative theory, are inappropriate to what is, or ought to be, conceived of and practised as a semantic activity within which certain techniques drawn from the plan ning discipline may be appropriate. New approaches to both planning and implementation are required to facilitate the communication of messages between planners and clients. These messages will contain much more than the mere detail of plan objectives and implementing requirements. They will contain information about the social, cultural, political and economic needs of all the parties engaged in the transaction. I have indicated that as presently practised the bureaucratic and technical assumptions vest power in the planners' views and opinions. This produces an asymmetrical relationship between planner and client. To rectify this tendency, we need to consider the problem of the appropriate forms of social organization; and the problem of balance between 'technique', the planner's perceptual apparatus which is essentially exterior, general and foreign to the client's perception, and may in addition be quantitative or algebraic, and the perception of the consumer, which is more likely to be qualitative and local. Mannheim distinguished between a 'radius of action' and a 'radius of foresight" understanding by the former "the cxtent of the causal sequences directly brought about by our initial activity and remaining more or less under our control", and by the latter "the length of the causal chain that can be more or less accurately forecast in a given situation as regards this initial activity". It is the inter-communication of these for different sets of people in the planning and implementation process which might be one goal of an 'open' approach. The radius of action and the radius of foresight of the planner and the administrator are assumed in conventional planning approaches to be in some sense broader than those of the client. Of course, in terms of planning premises as to 'significance', this is true. It has to be. But if we shift our focus, and look at the world of the client, we see that in other respects his scope of action and foresight are themselves very wide.

\section{Diagram 1}

(a) planner $\longrightarrow$

(b) planners

\section{Diagram 2}

(a) plan objectives $\longrightarrow$

(b) plan objectives client/victims clicnts/victims non-participative planning and implementation participative planning and implementation
hierarchy reinforces 1 (a) plan achievements group social forms facilitate $1(\mathrm{~b}) \longrightarrow$ plan achievements

\footnotetext{
2 G. Ieech, Semantics, Penguin, Harmondsworth, 1974.

3 C. Ryecroft, Psychoanalysis Observed, Constable, London, 1964 .
}

4 Mannheim, Man and Society in the Age of Reconstruction, Harcourt, Brace and Werld Inc., New York, 1941. 
Once we accept that both client and planner have equal weight, we can move to a more symmetrical relationship. We discover that they have different views of action and foresight, but that one is not superior to the other. In adopting this perspective a theoretical resolution of the problem of shifting from 1(a) to 1(b) and from 2(a) to 2(b) in the diagrams on page 43 which summarize my argument so far is brought into view. But how would such a resolution look in practice?

Two problems arise. One is that of social organization, to which I have already drawn attention and to which I will return below. The other is the problem of what kind of things semantically inclined planners ought to be doing. An attempt to come to terms with this problem is found in the work of Robert Caillot, who argues that the mass of the population should be mobilized to draft a plan, not only to execute it. ${ }^{5}$ For Caillot it is understood that:

(i) problems which are concretely experienced are not reducible to any intellectual or ideological perspective anyone has of them;

(ii) the possession of knowledge confers on no-one, including planners and technical experts, the right to decide on behalf of others who are in their terms less knowledgeable;

(iii) no-one can possess more than a partial view of reality. ${ }^{6}$

These axioms lead him towards a planning process with the following elements: "One, definition and qualitative description of the structures which comprise development; two, geographic localization of these diverse structures; three, analysis of these structures, to a large extent by those who live within them; four, synthesis of all these elements. This effort is made in the presence of all participants; five, an operational phase which consists in transforming study groups into action teams."7

In this process, "In a certain sense, there is no longer any distinction between surveyors and the surveyed (one might add here between the planners and planned), there are only instigators and actors. The former helped to clarify the dimensions of problems, the latter exercise choices and made decisions, each one at his own level in professional or administrative life." 8

How are people to become effectively involved in such processes? The model which might begin to provide an answer to this is that used in personal

\footnotetext{
5 R. Caillot. 'L'Enquête--participation à èconomie et humanisme'. in Cahiers de l'Institut d'education des adultes, no. 3, February 1967.

6 Op. cit., p. 161

7 Ibid., p. 162.

$8 \mathrm{R}$. Cailot, Une Connaissance Engagée, p. 56, in Goulet, op. cit., p. 163
}

and group counselling, another area where 'the semantic' is more important than 'the logical'.9 Counsellors are trained to learn and understand the private emotional and intellectual language of their client, and to translate it into ordinary communicable language. Understanding is unlikely ever to be complete, but it is sufficient to enable clients to share their experience, understand it and gain more control over it. In no sense does counselling 'solve' problems; rather it makes sense of problems and puts them in a new perspective.

Objections might be raised that such methods are highly sophisticated, requiring not only Western education but also Western neurosis, and these precise techniques might not be directly transferable between cultures. This is not important: appropriate techniques could be developed. What is important is that the conscious mutual exploration of problems at other than the intellectual level through inter-personal sensitivity is a relatively recent development in the West. Indeed, it has been suggested that this approach is similar to, and influenced by, non-European traditions of psychology. ${ }^{10}$

In conclusion, I had better note some of the things I am not saying. I am not taking the naive position that if only people would communicate all would be well. The approach which I have outlined might serve to make conflicts clearer, and help alter the existing balances of power and privilege in societies, but that is another matter. Neither am I assuming that societies are historically and geographically isolated. In particular, the implications of dependency theory for this approach need to be worked out, as do many other aspects of its situation in a real political and economic context. What I am suggesting, perhaps more tentatively than $I$ have expressed it, is that if we understand planning differently we may perhaps be able to do it differently and perhaps more effectively, whoever 'we' may be.

9 For an introduction to these areas see: E. Venables, Counselling, National Marriage Guidance Council, London, 1971; C. R. Rogers, Client Centred Therapy, Houghton Mifflin \& Co., Boston, 1965.

10 S. Goonatilake, 'The Late Development Effect: Some Basic Difficulties in the Theory', IDS Bulletin, vol 6 , no. 3 , February 1975 .

\section{Erratum}

IDS Bulletin 7 (4), Dudley Seers "A New Look at the Three World Classification", second line from the bottom of the table on page 10 should read:

Cuba * $\quad$ * 0 * 